\title{
Modelling of Cancer Growth, Evolution and Invasion: Bridging Scales and Models ${ }^{1}$
}

\author{
A.R.A. Anderson ${ }^{a 23}$, K.A. Rejniak ${ }^{a 3}$, P. Gerlee ${ }^{a 3}$ and V. Quaranta ${ }^{b}$ \\ ${ }^{a}$ Division of Mathematics, University of Dundee, Dundee, Scotland, UK \\ ${ }^{b}$ Department of Cancer Biology, Vanderbilt University School of Medicine, Nashville, TN, USA
}

\begin{abstract}
Since cancer is a complex phenomenon that incorporates events occurring on different length and time scales, therefore multiscale models are needed if we hope to adequately address cancer specific questions. In this paper we present three different multiscale individual-cell-based models, each motivated by cancer-related problems emerging from each of the spatial scales: extracellular, cellular or subcellular, but also incorporating relevant information from other levels. We apply these hybrid models to investigate the influence of the microenvironement on tumour invasion, cell-cell collaboration and competition leading to the initiation and growth of epithelial tumours, and to evolution of cell phenotypes/genotypes arising in tumours growing in different oxygen concentrations. We also discuss how these models relate to one another and can be used to bridge biological scales relevant to cancer.
\end{abstract}

Key words: tumour growth, tumour evolution, tumour invasion, single-cell-based models, hybrid models, multiscale

AMS subject classification: 82B24, 92C15, 92C17, 92B20, 92D15, 76D05, 76Z99, 92C37, $92 \mathrm{C} 50$

\section{Introduction}

There is no doubt that cancer is a complex phenomenon that includes events occurring on different length and time scales. It is widely accepted that tumour growth starts from a single cell that

\footnotetext{
${ }^{1}$ This work was supported by the Integrative Cancer Biology Program of the U.S. National Cancer Institute (U54 CA113007).

${ }^{2}$ corresponding author: anderson@maths.dundee.ac.uk

${ }^{3}$ These authors contributed equally to this paper
} 
has accumulated certain genetic mutations leading to uncontrolled cell proliferation which in turn gives rise to a clone of similarly mutated cells. Such genetic mutations take place on a subcellular level but manifest themselves on a cellular level by allowing the mutated cells to gain some proliferative advantage over competing neighbouring cells. This in turn leads to functional and morphological changes at the extracellular level where a mass of growing tumour cells interacts with its microenvironment, invades the surrounding tissues and can metastasise to distant parts of the body. However, the selection pressure exerted by the microenvironment, whether it is the density of the surrounding matrix or the background nutrient concentration, may have an effect on the genetic signature of the invading tumour cells. Therefore, certain cancer-related events occurring on one scale can also affect or be affected by changes occurring on other scales which could constitute a feedback loop that bridges scales. For example, mutations at the gene level can lead to changes at the cellular level which modify the cells microenvironment, this subsequently initiates a selective pressure upon the cell genotype leading to a potentially different cellular phenotype.

The whole process of tumour development includes several distinct stages that are initiated via the accumulation of genetic mutations leading to a cancerous cell. Subsequent avascular tumour growth leads to the initiation of angiogenesis (the outgrowth of tissue vasculature in response to stimuli produced by a dormant tumour) and vascularisation of the tumour. Once vascularised the tumour now has the potential to metastasis, but needs to first breach the basment membrane and invade the surrounding tissues, then intravasate (breaching the vessel walls to penetrate into a blood or lymph vessel). Passive transport through the lymph or blood vessels, subsequent extravasation (penetrating the vessel walls to leave the blood or lymph system) can lead to the formation of secondary tumours in distant parts of the body, [1]. We will consider here only the initial stages of tumour development - its initiation and avascular growth - in order to illustrate how mathematical models can be designed to address particular cancer-related questions and how separate models can bridge different biological scales and can be linked together to gain more understanding of the problems under consideration.

To address problems related to the initiation of tumour growth, its progression and invasion, mathematical models are needed that account for heterogeneity of both the tumour tissue and the surrounding environment. These models should crucially incorporate information from different biological scales, such as genetic changes, cell membrane protein redistribution, mechanics of cell life processes, kinetics of nutrient uptake, cell migration and adhesion. Over the last few years many mathematical models of tumour growth, both temporal and spatio-temporal, have appeared in the research literature (see [2] and [3] for a review). Deterministic reaction-diffusion equations have been used to model the spatial spread of tumours in the form of invading travelling waves of cancer cells $[4,5,6,7,8,9]$. Other mathematical techniques used to model the cumulative growth of tumours are based on boundary-integral, finite-element and level-set methods [10, 11]. Whilst all these models are able to capture the tumour structure at the tissue level, they fail to describe the tumour at the cellular level. On the other hand, single-cell-based models provide such a description and allow for a more realistic stochastic approach at both the cellular and subcellular levels. Several different discrete models of tumour growth have been developed recently, including cellular automata models $[12,13,14,15,16,17,18]$, Potts models $[19,20,21]$, lattice free cellcentered models [22] or agent-based models [23]. For a review on many different single-cell-based 
models applied to tumour growth and other biological problems see [24].

We present here three examples of such multiscale single-cell-based models which have been motivated by cancer-related problems defined on specific biological scales: extracellular, cellular and subcellular. Even though they are defined on a specific scale they still incorporate relevant information from other scales. The structure of the paper is as follows: In section 2. we discuss a Hybrid Discrete Continuum (HDC) model that allows us to address questions concerning cellcell and cell-stroma interactions from the point view of the whole tissue (extracellular level). In section 3. we present an Immersed Boundary Cell (IBCell) model that enables an investigation of direct interactions between neighbouring normal and tumour cells (cellular scale) on the level of cell membrane receptors. In section 4. we describe an Evolutionary Hybrid Cellular Automata (EHCA) model that is a hybrid cellular automaton in which each cell is equipped with a feedforward artificial neural network that allows for an investigation of the cell genotype-phenotype mapping (subcellular level). We finish with a discussion on bridging different biological scales using multiscale models and on bridging different models across distinct biological scales in section 5.

\section{Extracellular scale-Hybrid Discrete-Continuum Model}

To address questions arising on the extracellular scale, such as how the microenvironment influences the growth of the whole tumour tissue that may contain millions of cells, how the direct cellstroma interactions lead to the emergence of tumour tissue invasion or whether the tumour acquires aggressive behaviour in a given microenvironmental setting we have developed the Hybrid Discrete Continuum (HDC) model. This model couples a continuum deterministic model of microenvironmental variables (based on a system of reaction-diffusion-chemotaxis equations) and a discrete cellular automata like model of individual tumour cell migration and interaction (based on a biased random-walk model). As we consider the cells as individuals this enables us to model specific cell traits (such as cell proliferation, death, cell-cell adhesion, mutation, and production/degradation of micorenvironment specific components) at the level of the individual cell, but since we are able to include in the model a large number of cells, this allows us to trace the behaviour of the whole tumour tissue. A detailed discussion on the types of system that this technique is applicable to is given in [28]. Applications of the technique can be found in [25, 26, 27, 28, 29, 30, 31].

\subsection{HDC model of tumour invasion}

We will base our mathematical model on generic solid tumour growth and focus on four key variables involved in tumour cell invasion, namely; tumour cell density (denoted by $n$ ), matrix degrading enzyme (MDE) concentration (denoted by $m$ ), ECM concentration (denoted by $f$ ) and oxygen concentration (denoted by $c$ ). We will only consider a two dimensional slice through a generic three dimensional tumour, one cell diameter thick, although extension to a full three dimensional model is straightforward [32]. The following system of coupled nonlinear partial differential equations describes the dynamics of the tumour cells, ECM, MDEs and oxygen, 


$$
\begin{aligned}
& \frac{\partial n}{\partial t}=\overbrace{d_{n} \nabla^{2} n}^{\text {random motility }}-\overbrace{\rho \nabla \cdot(n \nabla f)}^{\text {haptotaxis }}, \\
& \frac{\partial f}{\partial t}=-\overbrace{\eta m f}^{\text {degradation }}, \\
& \frac{\partial m}{\partial t}=\overbrace{d_{m} \nabla^{2} m}^{\text {diffusion }}+\overbrace{\frac{\kappa n}{\text { production }}-\overbrace{\sigma m}^{\text {decay }},}^{\text {diffusion }}, \overbrace{d_{c} \nabla^{2} c}^{\text {production }}+\overbrace{\nu f}^{\text {uptake }}-\overbrace{\omega n}^{\text {decay }}-\overbrace{\phi c}^{\partial t},
\end{aligned}
$$

were $d_{n}, d_{m}$ and $d_{c}$ are the tumour cell, MDE and oxygen diffusion coefficients respectively, $\rho$ the haptotaxis coefficient and $\eta, \kappa, \sigma, \nu, \omega$ and $\phi$ are positive constants. We should also note that cell-matrix adhesion is modelled here by the use of haptotaxis in the cell equation i.e. directed movement up gradients of ECM. Therefore $\rho$ may be considered as relating to the strength of the cell-matrix adhesion. Since this model has already been published [30] we will not discuss its derivation here. We assume that the ECM, oxygen, tumour cells and consequently the MDEs, remain within the domain of tissue under consideration and therefore no-flux boundary conditions are imposed.

The HDC technique will be used to model the individual tumour cells and their interactions with one another and the continuum variables in the system (2.1). This first involves discretising (using standard finite-difference methods) the system of partial differential equations (2.1). We then use the resulting coefficients of the finite-difference stencil to generate the probabilities of movement of an individual cell in response to its microenvironment, further details can be found in $[28,30]$. As an illustration of the technique we only consider the tumour cell equation and discretise (2.1) in two spatial dimensions using central finite difference approximations to obtain the following,

$$
n_{i, j}^{q+1}=n_{i, j}^{q} P_{0}+n_{i+1, j}^{q} P_{1}+n_{i-1, j}^{q} P_{2}+n_{i, j+1}^{q} P_{3}+n_{i, j-1}^{q} P_{4}
$$

where the subscripts specify the location on the lattice and the superscripts the time steps. That is $x=i \Delta x, y=j \Delta x$ and $t=q \Delta t$ where $i, j, \Delta t, q$ and $\Delta x$ are positive parameters.

The central assumption of the HDC technique is that the five coefficients $P_{0}$ to $P_{4}$ from (2.2) can be thought of as being proportional to the probabilities of a cell being stationary or moving to one of four orthogonal neighbours. The coefficient $P_{0}$, which is proportional to the probability of no movement, has the form,

$$
P_{0}=1-\frac{4 \Delta t d_{n}}{\Delta x^{2}}-\frac{\Delta t \rho}{\Delta x^{2}}\left(f_{i+1, j}^{q}+f_{i-1, j}^{q}-4 f_{i, j}^{q}+f_{i, j+1}^{q}+f_{i, j-1}^{q}\right),
$$

and the coefficients $P_{1}, P_{2}, P_{3}$ and $P_{4}$, which are proportional to the probabilities of moving west, east, south and north respectively, have the forms,

$$
P_{1}=\frac{\Delta t d_{n}}{\Delta x^{2}}-\frac{\Delta t \rho}{4 \Delta x^{2}}\left[f_{i+1, j}^{q}-f_{i-1, j}^{q}\right], P_{2}=\frac{\Delta t d_{n}}{\Delta x^{2}}+\frac{\Delta t \rho}{4 \Delta x^{2}}\left[f_{i+1, j}^{q}-f_{i-1, j}^{q}\right],
$$




$$
P_{3}=\frac{\Delta t d_{n}}{\Delta x^{2}}-\frac{\Delta t \rho}{4 \Delta x^{2}}\left[f_{i, j+1}^{q}-f_{i, j-1}^{q}\right], P_{4}=\frac{\Delta t d_{n}}{\Delta x^{2}}+\frac{\Delta t \rho}{4 \Delta x^{2}}\left[f_{i, j+1}^{q}-f_{i, j-1}^{q}\right] .
$$

where the subscripts specify the location on the grid and the superscripts the time steps and $i, j$, $\Delta t, q$ and $\Delta x$ are positive parameters. From the above probabilities we see that if there were no ECM the values of $P_{1}$ to $P_{4}$ would be equal, with $P_{0}$ smaller (or larger, depending on the precise values chosen for the space and time steps) i.e. there is no bias in any one direction and the tumour cell is less (more) likely to be stationary - approximating an unbiased random walk. However, if there are gradients in the ECM, haptotaxis contributes to the migration process and the coefficients $P_{0}$ to $P_{4}$ will become biased towards the direction of increased ECM concentration. The motion of an individual cell is therefore governed by its interactions with its local ECM environment but will also be modified by interactions with other tumour cells.

Tumour heterogeneity at the genetic level is well known and the so called "Guardian of the Genome", the p53 gene is widely considered as a precursor to much wider genetic variation [41]. Loss of p53 function (e.g. through mutation) allows for the propagation of damaged DNA to daughter cells [41]. Once the p53 mutation occurs many more mutations can easily accrue, these changes in the tumour cell genotype ultimately express themselves as behavioural changes in cell phenotype. In order to capture the process of mutation we assign cells a small probability $\left(P_{\text {mutat }}\right)$ of changing their phenotype during cell division. If such a change occurs the cell is assigned a new phenotype from a pool of 100 randomly predefined phenotypes within a biologically relevant range of cell specific traits.

The simulation space therefore consists of a two dimensional lattice of the extracellular matrix $(\mathrm{ECM})(f)$ upon which oxygen $(c)$ diffuses and is produced/consumed, a matrix degrading enzyme $(m)$ diffuses and is produced by each of the individual tumour cells $\left(N_{i, j}\right)$ which occupy single lattice points $(i, j)$ and is assumed to degrade the ECM upon contact (see Fig. 1). Each cell will also have a set of predefined phenotypic traits including adhesion; proliferation; degradation and migration rates, which determines how it behaves and interacts with other cells and its environment.

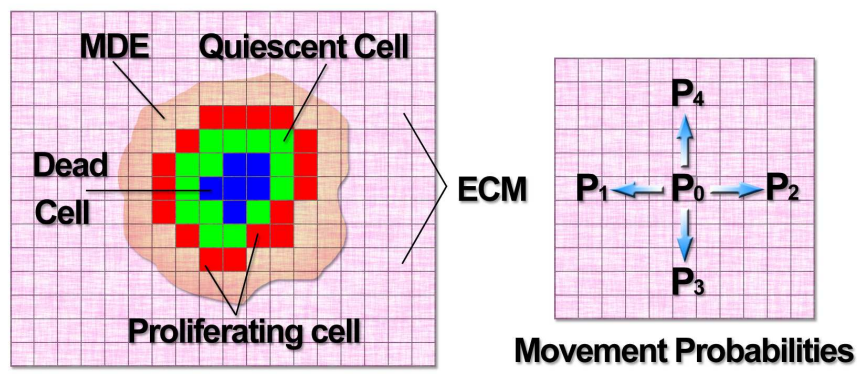

Figure 1: Schematic diagram (left) showing the key variables involved in the computational model: Tumour cells (colouration denoting cell state), extracellular matrix (ECM), matrix degrading enzyme (MDE). Oxygen production comes from a pre-existing blood supply that is proportional to the ECM density. Diagram (right) shows the four possible directions each cell can move on the grid, driven by the movement probabilities $P_{0}$ to $P_{4}$, each cell can move at most one grid point at a time. 


\section{Individual-Based Processes}

In addition to having the ability to move each cell has a collection traits that define its phenotype and describe how the cell will behave. The specific traits we consider are proliferation age, oxygen consumption, MDE production, haptotaxis coefficient and cell-cell adhesion. We have chosen these specific traits based on the current views of the invasive phenotype [42]. In the following paragraphs we discuss some of the key processes and traits involved in the HDC model of invasion (see [30] for a more detailed discussion).

Cell-Cell Adhesion: To model cell-cell adhesion explicitly we assume each cell has its own internal adhesion value $\left(A_{i}\right)$ i.e. the number of neighbours that it will preferentially adhere to. We therefore examine the number of external neighbours each cell has $\left(A_{e}\right)$ and if $A_{e} \geq A_{i}$ then the cell is allowed to migrate, otherwise it remains stationary.

Necrosis: For a tumour cell to survive it requires sufficient oxygen, since some tumour cells have been found to survive in very poorly oxygenated environments, we make the assumption that the concentration has to drop to 0.05 non-dimensional units (where 1 would be the initial concentration) for cell death to occur. This assumption is also applied to quiescent tumour cells. The space that dead cells occupy becomes available to new cells as soon as they die.

Proliferation: An individual cell will produce two daughter cells as a result of mitosis provided: (i) the parent cell has reached maturity (see mutation paragraph) and (ii) there is sufficient space surrounding the parent cell for the two new daughter cells to occupy. In order to satisfy condition (ii), we assumed that one daughter cell replaces the parent cell and the other daughter cell will move to any one of the parent cell's four orthogonal neighbours that is empty. If no empty neighbours exist then the cell becomes quiescent and proliferation is delayed until space becomes available. Quiescent cells are assumed consume half the oxygen of normal cells.

Production/Degradation: Since we are modelling individual tumour cells we must consider production and consumption of MDE and oxygen at the level of a single cell. In the continuum model Eq.(2.1) we have these rates as being proportional to the tumour cell density. Now these terms will only be active at a specific lattice point if a tumour cell is occupying that point (i.e. we take $n=1$ ) otherwise they will be zero (i.e. we take $n=0$ ).

Mutation: Here we shall consider 100 randomly defined phenotypes, each phenotype has an equal probability of being selected. A cells phenotype consists of a randomly selected, proliferation age $\left(\right.$ Phen $\left._{\text {age }}=8-16 h r s\right), O_{2}$ consumption $\left(\right.$ Phen $\left._{O_{2}}=\omega-4 \omega\right)$, MDE production $\left(\right.$ Phen $_{m d e}=$ $\kappa-4 \kappa)$, haptotaxis coefficient $\left(\right.$ Phen $\left._{\text {taxis }}=\rho-4 \rho\right)$ and adhesion value $\left(\right.$ Phen $\left._{A}=0-3\right)$. In most cases the range of values each parameter can take to define the phenotype were chosen to represent biologically realistic limits. Each cell is initially assigned the values of one of the hundred randomly selected phenotypes and for each subsequent proliferation there is a small probability $\left(P_{\text {mutat }}\right)$ of further mutations occurring which will lead to another randomly selected phenotype and so on.

\section{Simulation Process for the Hybrid Discrete-Continuum Model}

Each time step of the simulation process involves solving the discrete form of the system (2.1) 
numerically to generate the five coefficients $P_{0}$ to $P_{4}$. We then normalise these coefficients to obtain five corresponding final probabilities of motion, where normalisation simply means division by the total of the five coefficients. Probability ranges are then computed by summing the coefficients to produce 5 ranges, we then generate a random number between 0 and 1 , and depending on the range which this number falls in, the current individual tumour cell under consideration will remain stationary or move to one of its orthogonal neighbours at each time step. This is provided the conditions imposed by cell-cell adhesion are satisfied. Once movement has occurred then the other individual based processes are considered and may result in cell death or proliferation and mutation.

\subsection{Application to microenvironment induced invasion}

The importance of the tumour microenvironment is currently of great interest to both the biological and the modelling communities. In particular, both the immediate microenvironment (cell-cell or cell-matrix interactions) and the extended microenvironment (e.g. vascular bed) are thought to play crucial roles in both tumour progression and suppression (see the recent series of papers in Nature Reviews Cancer for further detail, [43, 44, 45]). Aggressive tumours are often described as having an invasive phenotype, characterised by fingering margins as opposed to more benign tumours which are characterised by smooth non-invasive margins. Recently it has been shown that not only can the microevironment promote tumour progression but it can also drive the invasive tumour phenotype. The work of Weaver [46] focuses on the impact of tissue tension in driving the invasive phenotype and clearly correlates higher tension in the tissue (a harsher environment) with an invasive phenotype. Similarly Pennacchietti [47] has shown a relationship between a hypoxic tumour microenvironment (again a harsher environment) and the invasive phenotype.

We shall use the HDC model to examine the impact of three different microenvironments (i.e. homogeneous tissue, heterogeneous tissue, low nutrient concentrations) on both the tumour morphology and phenotypic heterogeneity and how these different microenvironments might enhance or induce invasive behaviour. The three different microenvironments we specifically consider are (A) homogeneous ECM $(f(x, y)=1)$, (B) random ECM $(0 \leq f(x, y) \leq 1)$ and (C) low oxygen $(c(x, y)=0.25)$. We will consider a spatial domain of $1 \mathrm{~cm}^{2}$ and a time scale of $16 \mathrm{hrs}$. The same non-dimensional parameter values that are used in the following three simulations are $d_{n}=0.0005$, $d_{m}=0.0005, d_{c}=0.5, d_{c_{c e l l}}=0.25, \rho=0.01, \eta=50, \kappa=1, \sigma=0, \nu=0.5, \omega=0.57$ and $\phi=0.025$. We also take the phenotype mutation probability to be $P_{\text {mutat }}=0.1$. We initiate 50 tumour cells in the centre of the domain each with a randomly assigned phenotype (from the 100 predefined at the start of the simulation, see Mutation paragraph above). After 200 time steps the tumour cell distributions along with their respective phenotype distribution were gathered to produce Fig. 2.

From the upper row of Fig. 2 we can see that the three different microenvironments have produced distinct tumour morphologies. In particular, the homogeneous ECM distribution has produced the largest most circular looking tumour (Fig. 2A) containing mainly dead inner cells and a thin rim of proliferating cells. The tumour that grew in the random ECM also has a dead inner core with a thin rim of proliferating cells, however, the branched fingered morphology is very 

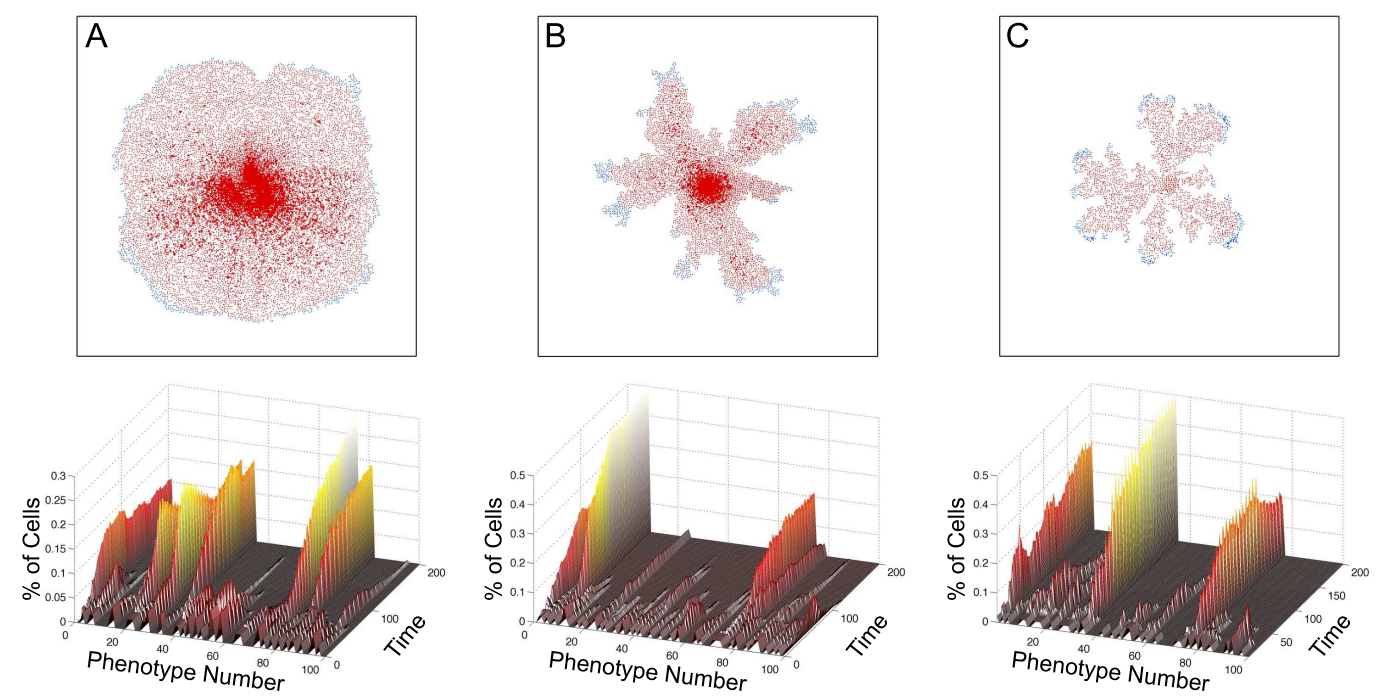

Figure 2: HDC tumour simulation results, at time $t=200$ units, using three different microenvironment conditions: (upper row) showing the tumour cell distributions at the end of simulated growth in (A) homogeneous ECM, (B) random ECM and (C) low oxygen (colouration denoting cell state: red [proliferating]; blue [necrotic]); (lower row) phenotype distributions for each of the respective tumour cell populations in the upper row.

different (Fig.2B). This fingering morphology is also observed in the low oxygen simulation which produced the smallest tumour again displaying the dead inner core with proliferating rim (Fig.2C). One obvious conclusion from these results is that by making the microenvironment more difficult (harsher) for the tumour to invade into, a fingered tumour displaying an invasive morphology results, Fig.2 B-C.

One of the major advantages of working with a computational model is the ability to keep track of all variables/paramters at all times. This allows us to examine the evolution of the tumour phenotype distribution, lower row of Fig.2, as the tumour invaded each of the different microenvironments. We note that there are approximately 6 phenotypes in the homogenous tumour, 2 phenotypes in the random tumour and 3 in the low oxygen tumour that dominate the tumour population and survive for most of the simulation. Of these phenotypes all have a zero cell-cell adhesion value, most have a short proliferation age, as well as high haptotaxis coefficients. Surprisingly, for each of the phenotypes that are selected, one in each of this subpopulation is the most aggressive phenotype, always being expressed by the largest fraction of cells in the tumour population, and always has the shortest proliferation age, highest haptotaxis coefficient, and no cell-cell adhesion restrictions.

The implication of these results is that an invasive outcome appears to be co-dependent upon the appropriate cell phenotype in combination with the right kind of microenvironment. In this case the phenotype is an aggressive one and the microenvironment is a harsh one. The cooperation, or more accurately the competition, that these two invasive properties confer upon the tumour population create a fingered invasive tumour. These results clearly have important clinical implications, since 
almost all current tumour treatments involve making the tumour microenvironment harsher (e.g. radiotherapy, chemotherapy, antiangiogenesis therapy). The tumour microenviroment should play an active role in treatment and not be considered as an innocent bystander which is currently the case. One possibility would be to combine treatments that simultaneously suppress the selective influence of the microenvironment and at the same time attack the tumour population. Whilst this is probably very difficult to achieve using current treatment protocols, it seems likely that as the crucial role of the microenvironment is unravelled such stratagies will become a reality.

\section{Cellular scale-Immersed Boundary Cell Model}

To address questions of direct interactions between cells that are crucial for the maintenance of a normal tissue architecture and for cell-cell competitive or collaborative behaviours that lead to the initiation of a tumour-like growth we have designed an Immersed Boundary Cell (IBCell) model of a eukaryotic cell. This model couples the mechanics of individual deformable elastic cells with the viscous incompressible fluid in which the cells sit. The cells can interact with other cells and with their environment via a set of discrete membrane receptors located on the cell boundary. Moreover, cell boundary points are also used to determine sites of fluid transport during cell growth or death. The fluid flow is influenced by sources and sinks used to model transport of fluid across the membranes of growing and dying cells, as well as forces generated by the deforming elastic cell boundaries, while at the same time all elastic bodies move at the local fluid velocity. The immersed boundary method was first introduced by Peskin to model blood flow around heart valves [48, 49], and has since been successfully applied to many different problems involving fluid-structure interactions; compare [50, section 9]. The IBCell model has been used previously to investigate folding of the trophoblast tissue [33], development of early avascular tumours [34], emergence of tumour microregions [35], formation of different patterns of ductal carcinomas in situ [36], the emergence of finger-like invasive protrusions of tumour tissue due to cell competition for space and nutrients [40], and cell self-arrangement into an epithelial acinus [51, 52]. Other possible applications and a 3-dimensional extension of the model are discussed in [37]. We describe below the mathematical framework of the IBCell model and three applications showing how cell direct collaboration or competition leads either to normal tissue formation or to tumour-like growth.

\subsection{Model of a eukaryotic cell and cell life processes}

The eukaryotic cells are modelled here as 2-dimensional fully deformable elastic bodies that interact with their microenvironment consisting of neighbouring cells and the extracellular matrix. The structure of each individual cell includes an elastic plasma membrane modelled as a network of linear springs that defines cell shape and encloses the viscous incompressible fluid representing the cytoplasm and providing cell mass. These individual cells can interact with other cells and with the environment via a set of discrete membrane receptors located on the cell boundary. In particular, each point can be engaged in adhesion either with one of the neighbouring cells (adhesive receptor) or with the extracellular matrix (basal receptor). Moreover, it can be used to determine sites of fluid 
transport during cell growth or death (free receptors). Figure 3(a) shows a small cluster of adherent cells with adhesive, basal and free receptors distributed along cell boundaries. Separate cells are connected by adhesive links, that are modelled as springs acting between boundary points of two distinct cells if they are sufficiently close to one another. Several cellular processes, such as cell growth, division, apoptotic death and epithelial polarisation, can be modelled within this immersed boundary framework. All individual cells follow identically defined cell life processes, however, since cells interact with one another, the initiation and progression of some cellular processes may be a result of the cells' collaborative or competitive behaviour. The mathematical formulation of cell life processes that are crucial for the applications presented in this section are described below.

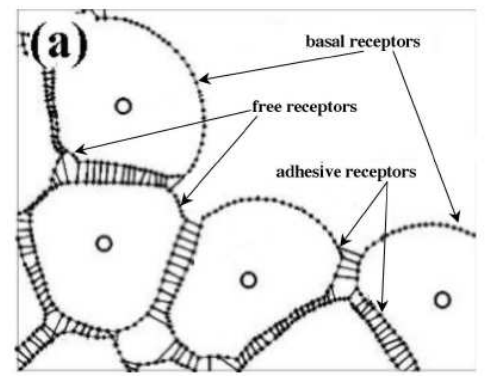

(b)
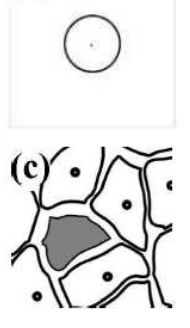
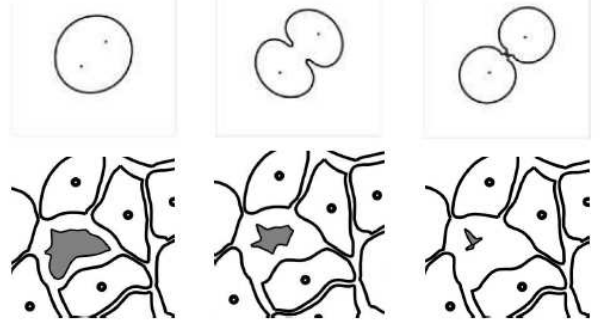

Figure 3: (a) A portion of a small cluster of adherent cells. Cell boundary points (dots) are connected by short linear springs to form cell membranes (grey lines); cell nuclei (circles) are located inside cells and are surrounded by cell cytoplasm modelled as a viscous incompressible Newtonian fluid; separate cells are connected by adherent links (thin black lines); basal, adhesive and free membrane receptors are distributed along the cell boundary; (b) Morphological alterations in a proliferating cell are presented in four consecutive snapshots that show: a cell ready to grow, an enlarged growing cell with two daughter nuclei, formation of the contractile ring orthogonal to the cell longest axis, cellular division into two daughter cells of approximately equal areas; (c) Morphological alterations in a cell dying by apoptosis (dark grey) located inside the cluster of viable cells are shown in four consecutive snapshots.

\section{Individual-Based Processes}

Cell proliferation: Cell growth is modelled by introducing fluid sources inside the host cell near the free membrane receptors and fluid sinks outside the cell boundary that creates a flow pushing on the boundary of the growing cell and increasing its area. The corresponding outgrowth of the cell membrane is modelled by introducing additional boundary points. The sources and sinks are subsequently deactivated when the cell area is doubled. Then, additional boundary forces are used to create the contractile ring splitting the host cell into two daughter cells of approximately equal areas. Figure 3(b) shows morphological changes in a single proliferating cell. It is assumed that the host cell is allowed to grow if at least $20 \%$ of its membrane receptors are free from cell-cell adhesive contacts. If the cell is too crowded by other cells to grow, it remains in a quiescent state until the space becomes available or the cell undergoes a differentiation process. 
Cell apoptosis: An apoptotic cell undergoes characteristic changes in its morphology, including detachment from the neighbouring cells, shrinkage of the cell volume, collapse of the cytoskeleton and alterations in the cell surface resulting in a bubbling appearance [53]. This process is modelled by a gradual shrinkage of the cell area due to release of the fluid to the outside environment. This is achieved first by disassembling all cell-cell adhesive contacts and by placing the sink-source couplets along the membrane of the whole cell with sinks located inside the cell and the balancing sources outside. The resulting fluid flow causes the cell boundary to collapse and the cell area is gradually reduced. It is assumed that the apoptotic cell is considered dead if its area has diminished to less than $15 \%$ of its initial value. Figure 3(c) shows morphological changes in a single cell dying by apoptosis (coloured in dark grey).

\section{Cell epithelial polarisation:}

A mature epithelial cell acquires three different types of membrane domain: an unbounded apical side facing free space inside the epithelium (lumen), a bounded side in direct contact with the extracellular matrix (a basal side), and a side adhering to membranes of other cells (a lateral surface), [54]. During the formation of an epithelial layer the cells must distinguish between these different domains in order to undergo the process of cell epithelial polarisation. This process can be modelled by inspecting the local microenvironments of all cell boundary receptors to recognise if they are either in contact with the extracellular matrix that constitutes the basal membrane domain, or in contact with other cells that forms the lateral membrane domain, or they are free from any cell-cell contacts that constitutes an apical domain.

\section{Governing equations:}

Equations (3.1)-(3.5) govern the motion of the fluid, of the immersed bodies and all interactions between the elastic cells and fluid.

The model is defined on a two-dimensional domain $\Omega$ with fixed Cartesian grid $\mathbf{x}=\left(x_{1}, x_{2}\right)$. The elastic membranes of all cells form a collection $\Gamma=\left\{\Gamma_{i}\right\}$ of closed curves $\Gamma_{i}$ defined in the curvilinear coordinates $\mathbf{X}_{i}(l, t)$, where $l$ is a position along the boundary of the $i^{t h}$ cell. The fluid inside and outside the cells is modelled as a homogeneous continuum with the same constant density $\rho$ and viscosity $\mu$. We assume that the fluid motion is governed by the Navier-Stokes equations, Eq.(3.1), where $p(\mathbf{x}, t)$ is the fluid pressure, $\mathbf{u}(\mathbf{x}, t)$ is the fluid velocity, the external force density $\mathbf{f}(\mathbf{x}, t)$ is defined at the boundaries of all cells, Eq.(3.3), and the source-sink distribution $s(\mathbf{x}, t)$ is taken around all growing or dying cells, Eq.(3.4). The continuity equation with a source term, Eq.(3.2), describes the law of mass balance, where the source distribution $s(\mathbf{x}, t)$ is identically equal to zero on the whole fluid domain except at the point sources and sinks that are used to model fluid transport in the growing or dying cells. Conservation of mass must be preserved globally in the fluid domain $\Omega$ at each time $t$; that is, $\int_{\Omega} s d \mathbf{x}=\rho \int_{\Omega}(\nabla \cdot \mathbf{u}) d \mathbf{x}=0$.

Interactions between the fluid and the material points of all immersed bodies are defined in Eq.(3.3)-(3.5). Here, the boundary forces $\mathbf{F}_{i}(l, t)$ in the $i^{t h}$ cell $\mathbf{X}_{i}(l, t)$ represent the sum of different contractive-repulsive forces arising from elasticity of the cell plasma membrane, division of the host cell into two daughter cells and from interactions between distinct cells. Each of them satisfies Hooke's law with a constant resting length $\mathcal{L}_{\alpha}$ and a constant spring stiffness $\mathcal{F}_{\alpha}$, where $\alpha$ denotes one of the acting forces described below. These forces are applied directly to the fluid in a 


$$
\begin{aligned}
& \rho\left(\frac{\partial \mathbf{u}(\mathbf{x}, t)}{\partial t}+(\mathbf{u}(\mathbf{x}, t) \cdot \nabla) \mathbf{u}(\mathbf{x}, t)\right)=-\nabla p(\mathbf{x}, t)+\mu \Delta \mathbf{u}(\mathbf{x}, t)+\frac{\mu}{3 \rho} \nabla s(\mathbf{x}, t)+\mathbf{f}(\mathbf{x}, t), \\
& \rho \nabla \cdot \mathbf{u}=s(\mathbf{x}, t), \\
& \mathbf{f}(\mathbf{x}, t)=\sum_{i} \mathbf{f}_{i}(\mathbf{x}, t) \quad \text { where } \quad \mathbf{f}_{i}(\mathbf{x}, t)=\int_{\Gamma_{i}} \mathbf{F}_{i}(l, t) \delta\left(\mathbf{x}-\mathbf{X}_{i}(l, t)\right) d l, \\
& \text { and } \quad \mathbf{F}_{i}(l, t)=\sum_{\alpha} \mathbf{F}_{\alpha(i)}(l, t), \\
& \text { and } \quad \mathbf{F}_{\alpha(i)}(l, t)=\mathcal{F}_{\alpha} \frac{\left\|\mathbf{X}_{i, k}(t)-\mathbf{X}_{i, l}(t)\right\|-\mathcal{L}_{\alpha}}{\left\|\mathbf{X}_{i, k}(t)-\mathbf{X}_{i, l}(t)\right\|}\left(\mathbf{X}_{i, k}(t)-\mathbf{X}_{i, l}(t)\right), \\
& s(\mathbf{x}, t)=\sum_{i} s_{i}(\mathbf{x}, t) \quad \text { where } \quad s_{i}(\mathbf{x}, t)=\sum_{k \in \Xi_{i}^{+}} S^{+}\left(\mathbf{Y}_{i, k}^{+}, t\right) \delta\left(\mathbf{x}-\mathbf{Y}_{i, k}^{+}\right)+\sum_{k \in \Xi_{i}^{-}} S^{-}\left(\mathbf{Y}_{i, k}^{-}, t\right) \delta\left(\mathbf{x}-\mathbf{Y}_{i, k}^{-}\right), \\
& \text {and } \sum_{k \in \Xi_{i}^{+}} S_{+}\left(\mathbf{Y}_{i, k}^{+}, t\right)+\sum_{m \in \Xi_{i}^{-}} S_{-}\left(\mathbf{Y}_{i, m}^{-}, t\right)=0 \text {. } \\
& \frac{\partial \mathbf{X}_{i}(l, t)}{\partial t}=\mathbf{u}\left(\mathbf{X}_{i}(l, t), t\right)=\int_{\Omega} \mathbf{u}(\mathbf{x}, t) \delta\left(\mathbf{x}-\mathbf{X}_{i}(l, t)\right) d \mathbf{x}
\end{aligned}
$$

$\delta$-layer region around the boundary $\Gamma_{i}$ of the $i^{\text {th }}$ cell, Eq.(3.3), where $\delta$ is a two-dimensional Dirac delta function: $\delta(\mathbf{x})=\delta\left(x_{1}\right) \delta\left(x_{2}\right)$.

The fluid sources $S^{+}\left(\mathbf{Y}_{i, k}^{+}, t\right)$ and sinks $S^{-}\left(\mathbf{Y}_{i, k}^{-}, t\right)$ are distributed at the discrete collections $\Xi_{i}^{p}$ of points $\mathbf{Y}_{i, k}^{p}$ (where $p=+$ or $p=-$, respectively), located along the cell boundary $\Gamma_{i}$ within the cell local microenvironment $\Theta_{\Gamma_{i}}=\bigcup_{\mathbf{X}_{i}(l, t) \in \Gamma_{i}}\left\{\mathbf{x}:\left\|\mathbf{x}-\mathbf{X}_{i}(l, t)\right\|<\varepsilon\right\}$. They are used to model transport of the fluid through the membranes of growing or dying cells in such a way, that fluid introduced in the sources $\mathbf{Y}_{i, k}^{+}$is balanced by the loss of fluid in the sinks $\mathbf{Y}_{i, k}^{-}$for each cell separately. These source and sink contributions are then transmitted to the surrounding fluid using the Dirac delta function, Eq.(3.4).

The no-slip condition of a viscous fluid implies that the material points on cell boundaries are carried along with the fluid, Eq.(3.5).

\section{Model implementation}

The model has been implemented using finite difference schemes for the fluid equations discretised on a regular square grid. The Navier-Stokes equations have been solved using the fast Fourier algorithm. Interactions between the fluid grid and the material points of immersed bodies have been implemented using a bell-shaped discrete approximation to the Dirac delta function of bounded (16-points) support. The general algorithm includes the following steps: (i) calculate the total force density from the configuration of cell boundaries; spread those values to the neighbouring grid points to determine the forces acting on the fluid; (ii) determine the source-sink distribution at points located along the boundaries of all growing and dying cells; spread these values to the neighbouring grid points to find the local expansion rate on the fluid; (iii) solve the Navier-Stokes 
equations for the fluid velocity field. (iv) interpolate the fluid velocity field to each immersed boundary point and compute their new positions by moving them at the local fluid velocity. More details about the IBCell model, its numerical implementation, computational solution and physical parameters can be found in $[33,34,35,37,51]$.

\subsection{Applications to tumour initiation and growth}

Direct collaborative or competitive interactions between neighbouring cells play a crucial role in the development and turnover of normal tissues. Failure to comply with such tightly controlled mechanisms may lead to tissue deformations or to uncontrolled tumour-like growth. We use the IBCell model to represent a 2-dimensional cross section through the central part of the mammary acinus, that is a 3-dimensional in vitro system resembling the structure of real cysts and glands. Mammary acini are also used to experimentally reconstruct some known phenotypes of epithelial tumours and to test the influence of various oncogenes on acinar growth and structure. We use the IBCell model to simulate the development of a hollow epithelial acinus and to determine cell-cell interactions leading to the formation of the hollow lumen inside the acinar structure and the surrounding layer of polarised cells. We then use this model to determine alteration in cell behaviour and cell-cell interactions that allow us to reproduce two kinds of epithelial tumours.

(i) 2

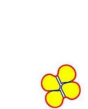

(ii)

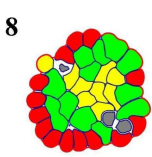

(iii)
4
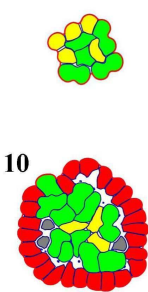

11

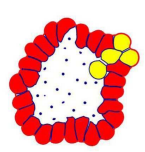

7
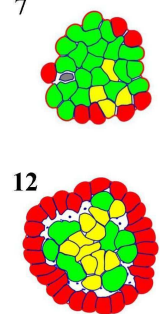

13.5

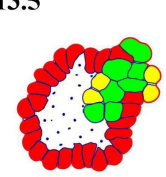

8
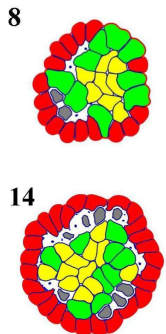

15.5

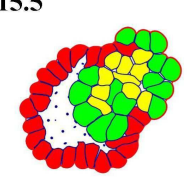

10
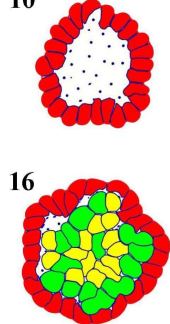

18.5

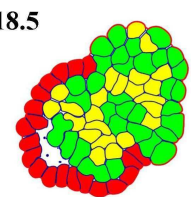

Figure 4: (i) Consecutive stages in the development of a normal hollow acinus from a small cluster of viable cells to an acinar structure composed of a layer of polarised cells surrounding the hollow lumen; (ii) Formation of a mutant acinus containing a layer of polarised cells and the lumenal space filled with cells emerging due to a failure in their growth suppression; (iii) Development of a mutant acinus with a cohort of invasive cells arising due to a failure in the polarisation of their predecessor. Time reported in the number of completed cell cycles. Cell phenotypes are coloured as follows: yellow-resting viable cells; green-growing cells; redpolarised cells; grey-apoptotic cells; black dots-dead cells.

Figure 4(i) shows consecutive stages in the development of a normal mammary acinus that 
arises from a small cluster of viable cells ( 2 nd cell cycle) that upon repeated divisions produces a larger cluster (4th cell cycle) consisting of several cells that self-organise into two subpopulations: outer cells having contact with the external medium and inner cells completely surrounded by other cells. Further development includes differentiation of outer cells into polarised cells that are in contact with the external medium (basal membrane domain), and have developed lateral membrane domains, i.e. tight adhesive connections with their neighbours (red cells, 7 th cell cycle). Subsequently, the polarised cells trigger the neighbouring inner cells to commit apoptotic death (grey cells, 8th cell cycle). Finally, all outer cells complete the polarisation process, whereas, all inner cells die by apoptosis (black dots, 10th cell cycle). Note, that due to adhesive connections between neighbouring polarised cells they acquire rectangular shapes.

The general algorithm defining changes in cell phenotypes during the development of the normal acinar structure can be summarised as follows: (i) a viable cell starts growing if at least $20 \%$ of its membrane receptors are free from cell-cell contacts, the process of cell growth is completed when the host cell doubles its area and then it starts dividing; (ii) if the cell is too crowded to start a growth process (i.e. when more than $80 \%$ of its membrane receptors are engaged in cell adhesion) the cell remains in a viable resting state until the space becomes available or the cell differentiates into a non-proliferating phenotype; (iii) when the cell develops the basal domain (i.e. at least $25 \%$ of cell membrane receptors are in contact with the extracellular matrix), and two lateral domains (i.e. the host cell adheres to two outer cells and each lateral side contains at least $10 \%$ of cell membrane receptors), the host cell acquires a basal-apical polarisation and creates an apical membrane domain by disconnecting all adherent links with the inner cells (as a result of this process the disconnected receptors of the inner cells are transformed into death receptors and are blocked from further cell-cell contacts and from sensing the free space in cell vicinity); (iv) the inner cells need to accumulate at least $25 \%$ of death receptors to start the process of apoptotic death that is completed when the cell area diminished to less than $15 \%$ of its initial value.

The failure to comply with some steps of this algorithm may result in the development of a tumour-like tissue. We present here two such cases. Figure 4(ii) shows several snapshots from a simulation of the growing acinus in which the outer cells develop a polarised phenotype in the very same way as in normal hollow acini, but the inner lumen remain partially filled with viable and growing cells, even if the apoptotic events are still present. In this simulation, the receptors of inner cells are not blocked upon an apoptotic death of their neighbours and thus these cells can sense free space released by the dying cells. This enables the newly born inner viable cells to start new proliferation processes that overcome still emerging apoptotic events. This failure in cell-cell interactions results in repopulation of the inner lumen by new viable cells that leads to a tumourlike filling of the lumenal space. Since the layer of polarised cells remains intact, this simulations resembles a ductal carcinoma in situ, a form of pre-invasive epithelial tumour. Figure 4(iii) shows an acinus in which one outer cell acquires a specific mutation that enables it to escape from the differentiated polarised state and to gain a proliferative advantage over its polarised neighbours (9.5th cell cycle). This leads to production of a small clone of identically mutated cells (11th cell cycle) that subsequently proliferate and expand (13.5th cell cycle) leading to a massive growth of a clone of aggressive cells that results in a tumour-like invasion into both the intraductal lumen and the extracellular space (15.5-18.5th cell cycle). Note, that all other ductal cells remain polarised. 
The examples in Figure 4 show the development of a normal hollow acinus and two tumourlike acinar structures - one corresponding to a pre-invasive ductal carcinoma in situ and the other to an invasive carcinoma. These three simulations differ only in the way some cell-cell interactions are defined. In the case of ductal carcinoma in situ the rules for cell polarisation and cell apoptosis are the same as in the case of the normal acinus, thus all outer cells acquire a polarised phenotype and the near-by inner cells are triggered to die by apoptosis. However, the growth of inner cells is not suppressed, thus whenever a free space is created by a dying cell it is subsequently used by the viable neighbours to initiate cell growth. The growing cells create adhesive connections with the polarised cells that in turn leads to new apoptotic events. Finally, almost all of the luminal space becomes repopulated by inner cells. Note, however, that the layer of epithelial cells remained intact and the overall shape of the acinar cluster has not changed significantly during this simulation. In the case of an invasive carcinoma it is assumed that the indicated cell and all its progeny lose their ability to polarise and gain ability to indefinite proliferation. Therefore, the clone of tumourlike cells that emerged from a single mutated cell is able to fill almost all of the luminal space and significantly spreads outside the acinus into the extracellular space. By changing interactions between different cell types we can simulate the formation of different normal and mutated tissue structures and we can test how changes in the properties of individual cells influence the structure and behaviour of the whole tissue.

\section{Subcellular scale-Evolutionary Hybrid Cellular Automata Model}

To address questions concerning the cell genotype-phenotype mapping, such as how the cell processes cues sensed from its vicinity and how its response depends on both the cell genotype and its microenvironment, we have designed an Evolutionary Hybrid Cellular Automata Model (EHCA) $[38,39]$. This model combines an artificial neural response network to model the cell decision mechanism (subcellular level) with a cellular automaton model to capture the behaviour of the whole tumour tissue. This model makes it possible to investigate why cells in similar conditions respond differently and how mutation in a single cell can impact the growth dynamics of the entire tumour. Each cell in our model is equipped with its own decision mechanism that makes the model different from previous cellular automaton models of tumour growth $[55,56,57]$ where cell behaviour was determined globally. This is crucial to capture an important aspect of clonal evolution, which is that cells within the tumour respond differently to their microenvironment.

\subsection{Evolutionary Hybrid Cellular Automata Model}

\section{The cell}

Each cell resides on a two dimensional lattice which represents a slice through the tissue under consideration and is equipped with a decision mechanism, which determines the behaviour of the cell based on the cell genotype and its local microenvironment. This decision mechanism is modelled 
using an artificial feed-forward neural network [58] in which the input vector $\xi=(n(\vec{x}, t), c(\vec{x}, t))$ contains the number of neighbours of the cell $n$ and the local oxygen concentration $c$, and the cell phenotype is determined by the network output representing the response for proliferation, quiescence and apoptosis. As these three behaviours are mutually exclusive the one with the strongest response is chosen, and we call it the life-cycle response. If the proliferation node has the strongest response the cell divides and produces a daughter cell, if the quiescence node has the strongest response the cell remains dormant and if the apoptosis node is strongest then the cell dies via apoptosis. The network modulates the metabolism of the cell depending on the life-cycle response. A cell in the proliferative state consumes oxygen at a rate $k_{0}$, while a quiescent cell has a lower consumption of $k_{q}<k_{0}$ and an apoptotic cell does not consume any oxygen.

The initial network, which is used as a "seed" in every simulation, is chosen so that the behaviour of the cell resembles that of an initial cancer cell phenotype. The response of the network therefore has to capture the essential behaviour of real cancer cells. The important features that we have included in the model are: (i) cells should perform apoptosis if the oxygen concentration $c(\vec{x}, t)$ falls below a certain threshold $h_{0}$ and (ii) cells should not divide if there is no space for the daughter cell (contact inhibition) i.e if $n(\vec{x}, t)>3$.

A graphical representation of the response network can be seen in Fig. 5(a). The output of the network is deterministic and depends only on the environmental input and the network parameters. These define the strength of connections between the different nodes and are represented as two matrices $w, W$ and two threshold vectors $\theta$ and $\phi$. When the cells divide the network parameters are copied to the daughter cell under mutations. The number of mutations that occur in the daughter cell network parameters is chosen from a Poisson distribution with parameter $p$. These mutations are then distributed equally over the matrices and the incorrect copying is modeled by adding a normal distributed number $s \in N(0, \sigma)$ to the daughter cell matrix or threshold entry, which means that $x \rightarrow x+s$, for those entries $x$ that are chosen for mutation. It should be noted that the mutation rate in this model does not correspond to the somatic mutation rate in human cells, as the amount of information copied by a real cell is approximately $10^{8}$ orders higher in magnitude. The mutations alter the connection strength between the nodes, which in turn changes how the cells responds to the microenvironment. If for example a mutation occurs in a connection that links the oxygen concentration with the apoptosis node this changes how the cell responds to the local oxygen concentration.

\section{Oxygen field}

The metabolism of cancer cells includes a large number of different chemicals that are all needed for maintenance and cell division, but it is known that oxygen availability limits the growth of the tumour [59]. Therefore, we focus here on how the oxygen impacts upon the growth and evolutionary dynamics of the model. To further simplify the situation we neglect the decay of the oxygen and assume that the oxygen is supplied from the boundary, which imitates the situation where the tissue is surrounded by blood vessels that supply the tumour with oxygen at a constant rate, similarly to $[56,55]$. Thus, the time evolution of the oxygen field is governed by the following partial differential equation, Eq. 4.1: 

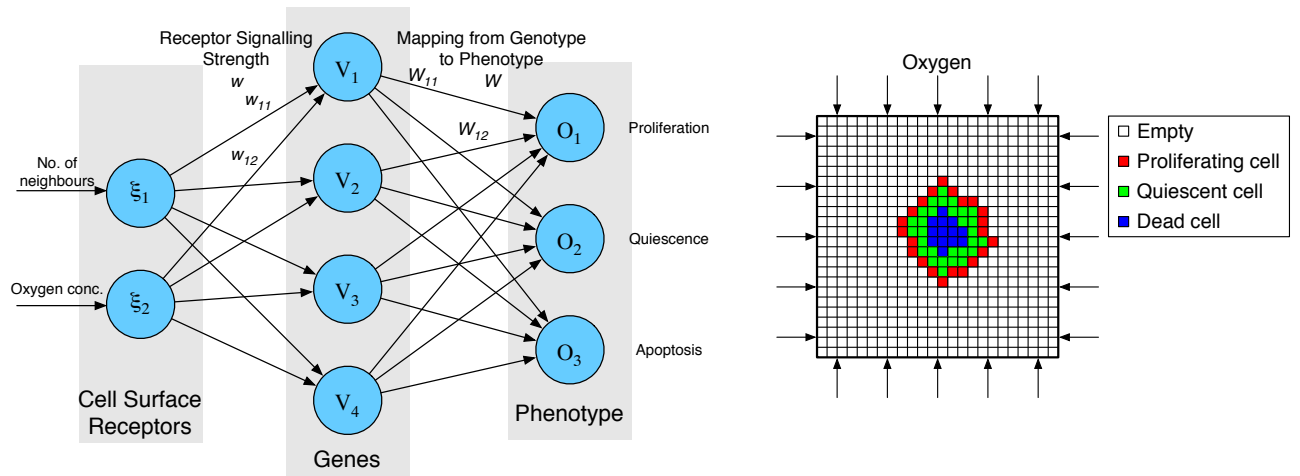

Figure 5: (a) The layout of the response network in the cells, the connections between the input layer and the hidden layer are determined by the connection matrix $w$ and the connections between the hidden layer and the output layer are determined by $W$. (b) Schematic layout of the model. The cells reside on a square lattice and each automaton element can either be occupied by a cancer cell or be empty. The cell type is indicated by the colour of the cell: Proliferating cells are coloured red, quiescent green and dead cells are blue. Coupled together with the discrete lattice is a continuous field of oxygen supplied from the boundary.

$$
\frac{\partial c(\vec{x}, t)}{\partial t}=D_{c} \nabla^{2} c(\vec{x}, t)-f_{c}(\vec{x}, t)
$$

with

$$
f_{c}(\vec{x}, t)= \begin{cases}0 & \begin{array}{l}
\text { if the automaton element at } \vec{x} \text { is empty, } \\
\text { i.e. no tumour cell at that lattice point } \\
F(\vec{x})
\end{array} \\
\text { if the automaton element is occupied, } \\
\text { i.e. a tumour cell exists at that lattice point }\end{cases}
$$

where $D_{c}$ is the diffusion constant of oxygen and the individual cell oxygen consumption rate $f_{c}(\vec{x}, t)$ depends on whether or not the automaton element at $\vec{x}$ is occupied by a tumour cell. $F(\vec{x})$ is equal to the oxygen consumption of the individual cell, which depends on the network response of the cell.

\section{Cellular automaton}

The two-dimensional tissue under consideration is represented by a $N \times N$ grid. Each grid point is either occupied by a cancer cell or is empty, and also holds the local concentration of oxygen, Fig. 5(b). The oxygen concentration interacts with the cells according to cellular consumption rates and are given appropriate initial and boundary conditions. Each time step the oxygen concentration is solved using a discretised form of equation (4.1) and every tumour cell is updated in a random order as follows: (i) The input vector $\xi$ is sampled from the local environment (i.e the grid point where the cell resides); (ii) A response $R=R(\xi, G)$ is calculated from the network, where $G$ represents the genotype of the cell, i.e. the network parameters; (iii) The cell consumes oxygen according to its behaviour. If there is not sufficient oxygen present the cell dies from necrosis; (iv) The life-cycle action determined by the network is carried out: (iv-a) If proliferation (P) is 
chosen, check if the cell has reached proliferation age and if there is space for a daughter cell. If both are true the cell divides and the daughter cell is placed in a neighbouring grid point, if not the cell does nothing; (iv-b) if quiescence (Q) is chosen the cell becomes quiescent; (iv-c) If apoptosis (A) is chosen the cell dies. If a cell dies from either apoptosis or necrosis it is no longer updated. Although the two death processes occur in different ways we will for simplicity treat them equally and consider the grid point where the cell resided empty in the next time step. For an in-depth description of the model and a full list of parameters see [38].

\subsection{Application to evolutionary dynamics of tumour growth}

The microenvironment and especially the oxygen concentration is known to influence the growth of solid tumours [59], the degree of hypoxia has been correlated with poor prognosis [60] and evolution towards aggressive phenotypes [61, 62] and invasive tumour morphology [63]. We will therefore use the EHCA model to investigate the impact of the oxygen concentration on both the morphology and evolutionary dynamics of tumour growth.

Fig. 6 shows the results of tumour growth simulation for two cases of the background oxygen concentration: (i) normal tissue concentration $c(\vec{x}, t)=c_{0}$ and (ii) poorly oxygenated tissue $c(\vec{x}, t)=c_{0} / 5$. Each simulation was started with an initial tumour of radius $R=5$ cells, each cell equipped with the same initial response network, and lasted for $t_{\max }=120$ time steps (80 days). In the normally oxygenated microenvironment the tumour starts growing with a rim of proliferating cells and a core of quiescent cells, but when the tumour reaches a critical size the oxygen supplied from the boundary is not sufficient and we observe the appearance of a necrotic core. From this point the tumour continues to grow, but now with a proliferating rim followed by an expanding region of dead cells. The growth dynamics in the low oxygen case on the other hand is quite different. In the beginning we still observe a similar structure, but after the appearance of cell death the dynamics diverge and a fingering growth pattern emerges, where the living cells reside on the tips of the fingers. It has been shown that for a simplified version of the model (without the evolutionary component) that the morphology of the tumour depends on how far the nutrient penetrates into the tumour [39]. For a wide proliferating rim (normal oxygen concentration) the growth is stable, while for a thin rim (low oxygen concentration) the tumour interface becomes unstable and this leads to branched growth.

In order to measure the change in behaviour or phenotype of the cells we have devised a measure on the response network that quantifies the behaviour of the cell. We do this by identifying each point in the two dimensional input space $\xi=(n, c)$ with the response it produces $R=(\mathcal{P}, \mathcal{Q}, \mathcal{A})$. In this way each response corresponds to a subset of the input space and we now measure the fraction of the input space that each of the corresponding responses occupy. This gives us a 3 -dimensional vector $S$, that we term the response vector, which reflects the behaviour of the cell. Formally we define three sets $x_{i}=\{\xi \in I ; R(\xi)=i\}$, where $R(\xi)$ is the network response to input vector $\xi, i=\mathcal{P}, \mathcal{Q}, \mathcal{A}$ and $I=[0,1] \times[0,4]$ is the set of all possible inputs to the network. 

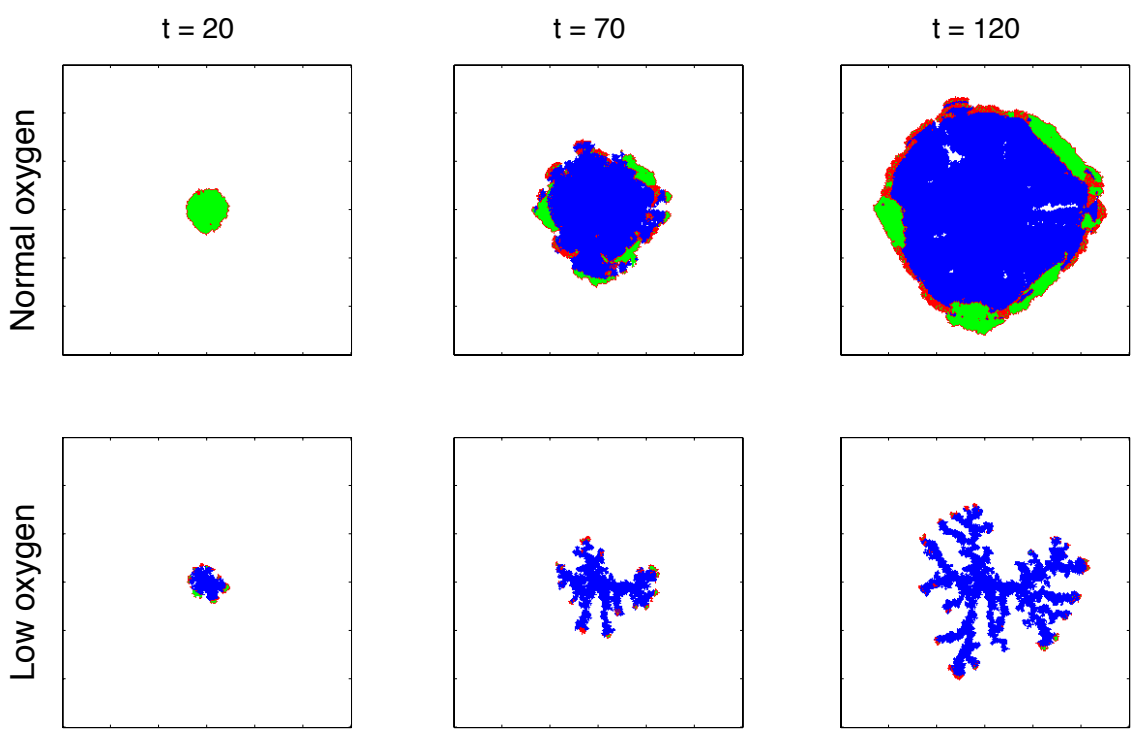

Figure 6: Spatial distribution of cells at $t=20,70$ and 120 for the normal and low oxygen concentration. Proliferating cells are red, quiescent cells are green and dead cells are blue.

The sizes of these subset are now given by,

$$
\left|x_{i}\right|=\frac{1}{B} \int_{I} \delta_{i, R(x)} d x
$$

where $\delta_{i j}$ is the Kronecker delta ( $\delta_{i j}=1$ if $i=j, 0$ otherwise) and $B=4$ is the area of the entire input space. The response vector can now be defined as $S=\left(\left|x_{P}\right|,\left|x_{Q}\right|,\left|x_{A}\right|\right)$. The initial genotype has a measure of $S=(0.67,0.18,0.15)$, which means that $67 \%$ of the input space corresponds to proliferation, $18 \%$ to quiescence and $15 \%$ to apoptosis. Note that this measure does not give any detailed information about the behaviour, but rather serves as a measure of the "average" behaviour of the cell or the potential the cell has for each response. This measure was used to analyse the phenotypic evolution in the population by calculating the average response vector $S_{a v g}$ over all cells in the population. The time evolution of $S_{\text {avg }}$ was tracked for both oxygen cases and is shown in Fig. 7a, where each entry in $S_{a v g}$ is plotted separately. In both environments we see evolution towards phenotypes with a larger proliferation potential and diminished response for quiescence and apoptosis. This is to be expected as only cells that are proliferating can spread their genetic material, however, we do see a difference between the two cases, where the phenotypic evolution is stronger in the low oxygen case.

The above measure reveals the dynamics on the phenotype scale, but the model also contains another level which is that of genotypes (we consider cells to have the same genotype if they have identical response networks). In order to investigate the dynamics on this scale we measured the 

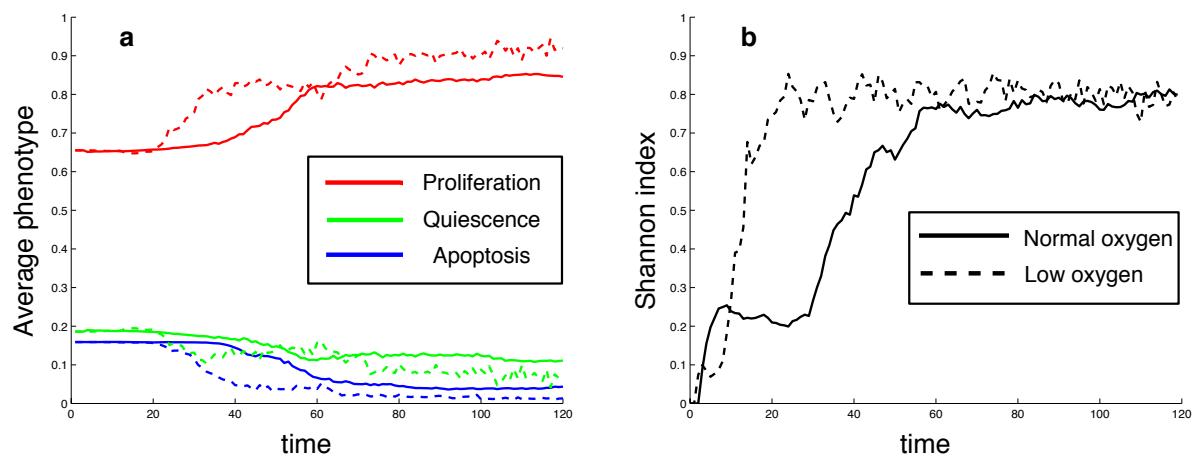

Figure 7: The time evolution of (a) the average phenotype $S_{\text {avg }}$ in the population and (b) the Shannon index of the population. In both plots the solid lines represents the results for the normal oxygen concentration while the dotted lines show the results for the low oxygen case.

Shannon index $H$ [64] or diversity of the genotype distribution given by,

$$
H=-\frac{1}{\ln (N)} \sum_{i=0}^{N}\left(p_{i} \ln p_{i}\right)
$$

where $p_{i}$ is the probability of finding subclone $i$ in the population and $N$ is the number of distinct subclones present in the population. The Shannon index reaches its maximum of 1 when all existing cells have different genotypes (i.e $p_{i}=\frac{1}{N}$ for all $i$ ), and its minimum 0 when the population consists of only one genotype. The time evolution of the Shannon index was measured for both oxygen concentrations and the result can be seen in Fig. $7 \mathrm{~b}$.

The low oxygen concentration also increases the competition between cells at the tumour boundary, as they have to compete for the limited oxygen supply. Cells with a small advantage will grow at the expense of their closest neighbours, and the low oxygen concentration can therefore be said to amplify both spatial and evolutionary perturbations. Spatial in the sense that a small protrusion on the tumour boundary will experience a higher flux of oxygen and therefore grow at a higher rate, and evolutionary in the sense that the selection pressure is stronger and a subclone with only a small growth advantage will be selected for due to the competition for oxygen. This is evident if we look at the evolution of the average phenotype in the two environments (Fig. 7a). In both cases we observe an evolution towards phenotypes with a larger potential to proliferate, but this phenomenon is more pronounced in the low oxygen case. In the beginning, before the tumour reaches its critical size and before the emergence of the necrotic core, both average phenotypes coincide. But as soon as oxygen becomes limited we see an increase in proliferative potential, which is directly related to the selection pressure that the limited supply of oxygen exerts. Although the supply of oxygen is limited in both cases, the lower concentration of oxygen means that the selective forces are stronger and that evolution towards proliferative phenotypes occurs both faster and is more notable. The increase in proliferation occurs at the expense of down-regulated 
quiescence and apoptosis, but from Fig. 7a we observe that apoptosis is more affected. This is in agreement with experimental results, which have shown that cancer cells from hypoxic tumours have a smaller apoptotic potential $[61,62]$. The hypoxic microenvironment provides a selection pressure for subclones that can withstand the low oxygen concentrations, which is exactly what we observe in our model.

The time evolution of the Shannon index reveals that the oxygen concentration also has an impact on the genotype dynamics (Fig. 7b). In the normal oxygen case we observe a slow increase in the genotype diversity, while in the low oxygen case this increase occurs much faster. Although the Shannon index settles around a similar value in the two cases $(H \approx 0.8)$, it clearly exhibits qualitatively different behaviours and again this can be attributed to the selection pressure that the oxygen concentration exerts. The early appearance of cell death in the low oxygen concentration makes it possible for subclones to exploit the new environment and consequently we observe an earlier increase in the population diversity. It should be noted that although phenotypic evolution in the low oxygen concentration is more directed towards aggressive phenotypes, the underlying genotypic distribution is more diverse than in the normal oxygen case. In conclusion we can observe that the oxygen concentration affects the dynamics on three different scales: the morphology of the tumour, the evolution of phenotypes and the genotype diversity.

Interestingly the low oxygen concentration that generates the selection pressure is due to the uncontrolled growth that occurs in tumours. This loss of homeostasis creates a situation where the expanding cancer cell population consumes more nutrients than can be supplied. This leads to a harsh microenvironment which selects for cells with for example reduced apoptotic potential. Due to their adaptation these cells will be able to survive and in doing so they will make the microenvironment even more deprived of nutrients. This creates a positive feedback where the microenvironment becomes increasingly hostile and the cancer cells even more adapted to harsh growth conditions. This is a situation which possibly could accelerate evolution towards more malignant and invasive phenotypes and could be responsible for the rapid progression of some tumours. Further it highlights the importance of taking into account the relation between microenvironment, cell genotype and phenotype when modelling the evolutionary dynamics of tumour growth.

In real cells the adaptation to hypoxic conditions occurs through mutations that affect subcellular pathways which in turn regulate cell behaviour. One example of this is the p53-gene, which in its wild-type form induces apoptosis in hypoxic conditions, while mutated forms of p53 lack this ability [65]. Although this model does not take into account the dynamics of these pathways it can still serve as a useful tool as it captures the dynamics on the scale where selection acts, i.e. on the level of cell behaviour. Of course in neglecting the exact dynamics of subcellular pathways we might disregard important mechanisms, but as the present knowledge of the subcellular machinery is at best partial it seems natural to focus on the relation between environmental input and cell phenotype. In this model this relation is captured by using a decision network whose parameters serve as the genetic material of the cell. This makes it possible to distinguish between the phenotype of the cell, which corresponds the output of the network, and the genotype, the network parameters. The phenotype is a function of the genotype, but also crucially depends on the microenvironment of the cell. Although it is still far from being an accurate model of the subcellular dynamics it 
brings the model closer to the real situation, and even in this simple set-up, where we only considered the oxygen concentration and the number of neighbours as input to the network, we can observe different dynamics on the phenotype and genotype level.

\section{Conclusions}

We have presented here three different single-cell-based models of tumour initiation, growth and invasion. Each of them was motivated by questions from different biological scales and varying complexity: extracellular, cellular or subcellular. Single-cell-based models allow for the introduction of heterogeneity within both tumour tissue and the surrounding environment. They enable one to treat each cell as an individual entity with individually regulated cell intrinsic properties, such as mutation, cell life processes, cell-cell and cell-stroma interactions. All three models represent a class of hybrid models that use both discrete and continuous variables. The cells are modelled in a discrete manner, whereas the environment (the extracellular matrix, the enzymes and nutrients) is described using a continuous approach. These models also incorporate information from different biological scales, such as genetic changes, protein redistribution, mechanics of cell life processes such as growth, death, polarisation and migration, kinetics of nutrient uptake, and cellcell or cell-stroma interactions. Fig. 8 shows schematically the elements from each of three basic biological scales (extracelluar, cellular and subcellular) that are incorporated into each of the three models discussed in this paper: a Hybrid Discrete Continuum (HDC), an Immersed Boundary Cell (IBCell) and an Evolutionary Hybrid Cellular Automata (EHCA). There is clearly much overlap between the three models and obviously there are many other components that could be included. It is probably worth discussing briefly the order in which these models were developed and what motived their implementation as it well help show how we have, to some extent, already formed real bridges between some of these models.

The HDC model focussed on the cell phenotype and was designed to address questions on the extracellular level, such as how the tumour is influenced by the microenvironment in which it grows, how tumour progression can differ depending on the structure and oxygenation of the surrounding stroma, and what kind of tissue environment will promote tumour invasiveness. However, this model lacked the ability to connect directly with much of the experimental cancer data currently being gathered on the genotype scale. This need led to the creation of the EHCA model that allows one to bridge the genotype and phenotype scales and yet contains much of the core of the HDC model. Therefore the EHCA model bridges what the HDC model could not do, but it does not represent the genotype in a precise or direct manner instead it does so by clustering genes into metagene clusters much in the same way that mircoarray analysis clusters different set of genes with cellular processes. The real power of this approach however will be realised when we can establish which real genes the metagene clusters contain. Another limitation with the HDC (and EHCA) model was the oversimplification of the cell-cell and cell-matrix adhesion processes. The need to better define the cell geometry and these processes as well as an understanding of what makes a normal tissue normal led to the development of the IBCell model. Detailed modelling of the cell geometry becomes crucially important when understanding how normal epithelial tissues 


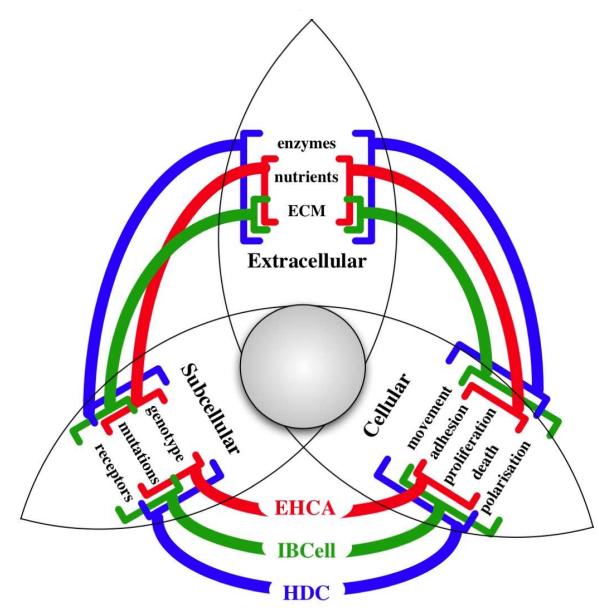

Figure 8: A schematic representation of bridging the biological scales: extracelluar, cellular and subcellular by three multiscale single-cell-based models: a Hybrid Discrete Continuum (HDC), an Immersed Boundary Cell (IBCell) and an Evolutionary Hybrid Cellular Automata (EHCA) applied to cancer-related problems.

are formed, as the orientation and shape of the cell are tightly integrated with the tissue formation. Understanding these processes can lead to a better knowledge of what can or may go wrong when a neoplastic lesion forms.

Both HDC and EHCA models use a cellular automata approach which can handle millions of cells, however, each cell is represented in a simplified way as a single square lattice site with four cell neighbours. In contrast, in the IBCell model all cells have variable shapes, are fully deformable, and interact with other cells via a set of cell membrane receptors. Moreover, the number of neighbouring cells is not pre-defined, depends only on cell shape and location within the tissue, and can dynamically change due to cell deformation whilst undergoing life processes. By introducing more detailed cell structure, however, the complexity of the computations increases, and thus the IBCell model works the best with up to a few hundred of cells.

In principle, it is possible to build a model that will contain a detailed description of cell morphology (such as in IBCell model), the genotype-phenotype apparatus (as in EHCA model), and will be able to handle large numbers of migrating and growing cells to faithfully represent a structure of the whole tumour tissue (such as in HDC and EHCA models), but such a model may begin to acquire structural complexity comparable with biological cells but be computationally far less effective than the real living organism. It would then be more desirable to find ways to bridge different independent models rather than build a model that encompasses all the complexity of tumour growth and invasion. In regards to cancer, the level of detail required and which scales should be bridged may be a function of the cancer development process itself. In the early stages of development one might want more specific detail about both the genetic and cellular components of the cell. But as the cells mutate and evolve this potentially leads to the dominance of a single subclone and then the need for genetic details maybe diminished. Also as the tumour 
population grows from hundreds to millions the detailed single cell geometry will become less important as the gross population morphology dominates and interactions with the stroma become more important.

Links between the IBCell model and the other two models have not yet directly been made but there are some obvious ways in which they could be interfaced. For instance, the IBCell approach can be used to model the initial tumour growth and the results can serve as an initial condition to the cellular automata class of models. The IBCell model can also be employed to investigate details of cell processes, such as the orientation of cell division that can be then used in the CAlike models to direct a position of newly born daughter cells instead of being chosen randomly. A detailed examination of cell-stroma interactions at the level of cell membrane receptors, such as consumption of nutrients, activation of latent matrix-related enzymes, and ECM degradation may be subsequently used to better define the biased motility of cells in CA models. On the other hand, the results of simulations of HDC and EHCA models in different environments and with different rules for cell mutation can define the dominant cell genotypic and phenotypic characteristics which may be used to directly define the mechanics of cell life processes, cell-cell and cell-stroma interactions in the IBCell model.

The EHCA model was mainly constructed to capture the genotype-phenotype relation and its impact on the evolutionary dynamics, but could also be extended with components from the other two models. For example the model treats receptors in a simplistic manner by only considering the number of neighbours and the oxygen concentration at one grid point as input to the decision network. This could easily be extended to a situation where the input nodes have a spatial location on the cell, by for example sensing the oxygen concentration in four directions and letting this influence the axis of cell division. Borrowing from a higher scale one could also introduce cell movement and making the cell phenotype dependent on ECM concentration, thus allowing for accurate modelling of tissue-scale dynamics.

One may want to conclude that by combining the best aspects of the different models into one and maybe adding a few more components, the definitive cancer model could be constructed. We argue that this is almost certainly not the case and its not simply due to computational constraints, it has more to do with the philosophy of modelling. Mathematical modelling of cancer is not something you can programme a machine to do automatically. Throwing large amounts of variables and processes together to build a highly complex cancer model, that may in fact produce simulations that "look realistic", will not ultimately produce a deeper understanding of the process itself. Mathematical modelling of cancer is rather an art that requires skill, thought, intuition and, crucially, dialogue with biologists, in order to distill the key variables that capture the essence of the cancer process under question. Placing these few crucial variables in the right modelling framework can produce novel insight into the fundamentals of the cancer process and naturally leads to experimentally testable hypotheses. Therefore, it is clear that the key lies really in the biological question that needs to be answered. This should drive the model derivation and define the scales at which the model operates and bridges. However, as questions become more complex models will need to do the same, but by linking several simpler well understood models, perhaps one can reduce this complexity to an understandable minimum. 


\section{References}

[1] J. P. Thiery. Epithelial-mesenchymal transition in tumours progression. Nature Reviews Cancer, 2 (2002), 442-447.

[2] R.P. Araujo, D.L.S. McElwain. A history of the study of solid tumour growth: the contribution of mathematical modeling. Bull. Math. Biol. 66 (2004), 1039-1091.

[3] L. Preziosi. Cancer modelling and simulation. CRC-Press, 2003.

[4] R.A. Gatenby, E.T. Gawlinski. A reaction-diffusion model of cancer invasion. Cancer Research, 56 (1996), 5745-5753.

[5] M.E. Orme, M.A.J. Chaplain. A mathematical model of vascular tumour growth and invasion. Mathl. Comp. Modelling, 23 (1996), 43-60.

[6] A.J. Perumpanani, J.A. Sherratt, J. Norbury, H.M. Byrne. Biological inferences from a mathematical model of malignant invasion. Invasion and Metastases, 16 (1996), 209-221.

[7] J.A. Sherratt, M.A. Nowak. Oncogenes, anti-oncogenes and the immune response to cancer: a mathematical model. Proc. R. Soc. Lond. B, 248 (1992), 261-271.

[8] K.R. Swanson, C. Bridge,J.D. Murray, E.C. Alvord Jr. Virtual and real brain tumors: using mathematical modeling to quantify glioma growth and invasion. J. Neuro. Sci., 216 (2003), $1-10$.

[9] J.P. Ward, J.R. King. Mathematical modelling of avascular-tumour growth II: modelling growth saturation. IMA J. Math. Appl. Med. Biol., 16 (1999), 171-211.

[10] X. Zheng, S.M. Wise, V. Cristini, (2005). Nonlinear simulation of tumour necrosis, neovascularization and tissue invasion via an adaptive finite-element/level-set method. Bull. Math. Biol. 67 (2005), 211-256.

[11] P. Macklin, J.S. Lowengrub. Evolving interfaces via gradients of geometry-dependent interior Poisson problems: application to tumor growth. J. Comput. Phys., 203 (2005), No. 1, 191220.

[12] T. Alarcon, H.M. Byrne, P.K. Maini. A multiple scale model for tumor growth. Multiscale Model Simul. 3 (2005), 440-475.

[13] S. Dormann, A. Deutsch. Modeling of self-organzied avascular tumor growth with a hybrid cellular automaton. In Silico Biology, 2 (2002), 0035.

[14] W. Düchting. Tumor growth simulation. Comput. \& Graphics, 14 (1990), 505-508.

[15] A.R. Kansal, S. Torquato, G.R. Harsh, E.A. Chiocca, T.S. Deisboeck. Simulated brain tumor growth using a three-dimensional cellular automaton. J. Theor. Biol. 203 (2000), 367-382. 
[16] A. Qi, X. Zheng, C. Du, B. An. A cellular automaton model of cancerous growth. J. theor. Biol., 161 (1993), 1-12.

[17] J. Smolle, H. Stettner. Computer simulation of tumour cell invasion by a stochastic growth model. J. theor. Biol., 160 (1993), 63-72.

[18] A. A. Patel, E. E. Gawlinski, S. K. Lemieux, R. A. Gatenby. A cellular automaton model of early tumor growth and invasion: the effects of native tissue vascularity and increased anaerobic tumor metabolism. J. Theoret. Biol., 213 (2001), 315-331.

[19] E. L. Scott, N. F. Britton, J. A. Glazier, M. Zajac. Stochastic simulation of benign avascular tumour growth using the Potts model. Mathematical and Computer Modelling, 30 (1999), 183-198.

[20] S. Turner, J. A. Sherratt. Intracellular adhesion and cancer invasion: a discrete simulation using the extended Potts model. J. theor. Biol, 216 (2002), 85-100.

[21] Y. Jiang, J. A. Pjesivac-Grbovic, C. Cantrell, J. P. Freyer. A multiscale model for avascular tumour growth. Biophys. J., 89 (2005), 3884-3894.

[22] D. Drasdo, S. Höhme. Individual-based approaches to birth and death in avascular tumors. Mathematical and Computer Modelling, 37 (2003), 1163-1175.

[23] L. Zhang, C.A. Athale, T.S. Deisboeck. Development of a three-dimensional multiscale agent-based tumor model: simulating gene-protein interaction profiles, cell phenotypes and multicellular patterns in brain cancer. J. Theo. Biol. 244 (2007), 96-107.

[24] A. R. A. Anderson, M. A. J. Chaplain, K. A. Rejniak. Single-cell-based models in biology and medicine. Birkhauser, Bassel, 2007.

[25] A. R. A. Anderson, B.D. Sleeman, I.M. Young, B.S. Griffiths. Nematode movement along a chemical gradient in a structurally heterogeneous environment: II. Theory. Fundam. appl. Nematol., 20 (1997), 165-172.

[26] A. R. A. Anderson, M. A. J. Chaplain. Continuous and discrete mathematical models of tumour-induced angiogenesis. Bull. Math. Biol., 60 (1998), 857-899.

[27] A. R. A. Anderson, M.A.J. Chaplain, E.L. Newman, R.J.C. Steele, A.M. Thompson. Mathematical modelling of tumour invasion and metastasis. J. Theoret. Med., 2 (2000), 129-154.

[28] A. R. A. Anderson. A hybrid discrete-continuum technique for individual based migration models. In: Polymer and Cell Dynamics. Eds. W. Alt, M. Chaplain, M. Griebel, J. Lenz. Birkhauser, Basel, 2003.

[29] A. R. A. Anderson, A. Pitcairn. Application of the hybrid discrete-continuum technique. In: Polymer and Cell Dynamics. Eds. W. Alt, M. Chaplain, M. Griebel, J. Lenz. Birkhauser, Basel, 2003. 
[30] A. R. A. Anderson. A hybrid mathematical model of solid tumour invasion: the importance of cell adhesion. IMA J. Math. Med. and Biol., 22 (2005), 163-186.

[31] A. R. A. Anderson, A.M. Weaver, P.T. Cummings, V. Quaranta. Tumor morphology and phenotypic evolution driven by selective pressure from the microenvironment. Cell, 127 (2006), 1-11.

[32] A. R. A. Anderson. A hybrid multiscale model of tumour invasion: evolution and the microenvironment. In: Single-Cell-Based Models in Biology and Medicine, eds. A. R. A. Anderson, M. A. J. Chaplain, K. A. Rejniak, 2007, Birkhauser: Basel.

[33] K. A. Rejniak, H. J. Kliman, L. J. Fauci. A computational model of the mechanics of growth of the villous trophoblast bilayer. Bull. Math. Biol., 66 (2004), 199-232.

[34] K. A. Rejniak. An immersed boundary framework for modelling the growth of individual cells: an application to the early tumour development. J. Theor. Biol., 247 (2007), 186-204.

[35] K. A. Rejniak. A single-cell approach in modeling the dynamics of tumor microregions. Math. Biosci. Eng. 2 (2005), 643-655.

[36] K. A. Rejniak, R. H. Dillon. A single cell based model of the ductal tumor microarchitecture. Comput. Math. Meth. Medic., 8 (2007), No. 1, 51-69.

[37] K. A. Rejniak. Modelling the development of complex tissues using individual viscoelastic cells. In: Anderson, A.R.A., Chaplain M.A.J., Rejniak K.A (ed.) Single-cell-based models in biology and medicine. Birkhauser-Verlag, 2007.

[38] P. Gerlee, A.R.A. Anderson, An evolutionary hybrid cellular automaton model of solid tumour growth. Journal of Theoretical Biology, 246 (2007), No. 4, 583-603.

[39] P. Gerlee, A.R.A. Anderson. Stability analysis of a hybrid cellular automaton model of cell colony growth. Physical Review E, 75 (2007), 051911.

[40] A. R. A. Anderson, K. A. Rejniak, P. Gerlee, V. Quaranta. Microenvironment driven invasion: a multiscale multimodel investigation. Journal of Mathematical Biology, in review.

[41] D.P. Lane. The regulation of p53 function. Steiner Award Lecture. Int. J. Cancer, 57 (1994), 623-627.

[42] D. Hanahan, R.A. Weinberg, R.A. (2000). The hallmarks of cancer. Cell, 100 (2000), 57-70.

[43] A. Albini, M.B. Sporn. The tumour microenvironment as a target for chemoprevention. Natrure Rev. Cancer, 7 (2007), doi:10.1038.

[44] B. Bierie, H.L. Moses. Tumour microenvironment: TGF: the molecular Jekyll and Hyde of cancer. Nature Rev. Cancer, 6 (2006), 506-520. 
[45] C.M. Overall, O. Kleifeld. Tumour microenvironment Opinion: validating matrix metalloproteinases as drug targets and anti-targets for cancer therapy. Nature Rev. Cancer, 6 (2006), 227-239.

[46] M.J. Paszek, N. Zahir, K.R. Johnson, J.N. Lakins, G.I. Rozenberg, A. Gefen, C.A. ReinhartKing, S.S. Margulies, M. Dembo, D. Boettiger, D.A. Hammer, V.M. Weaver. Tensional homeostasis and the malignant phenotype. Cancer Cell, 8 (2005), 241-254.

[47] S. Pennacchietti, P. Michieli, M. Galluzzo, M. Mazzone, S. Giordano, P.M. Comoglio. Hypoxia promotes invasive growth by transcriptional activation of the met protooncogene. Cancer Cell, 3 (2003), 347-361.

[48] C. S. Peskin. Flow patterns around heart valves: A numerical method. J. Comput. Phys., 10 (1972), 252-271.

[49] C. S. Peskin. Numerical analysis of blood flow in the heart. J. Comput. Phys., 25 (1977), 220-252.

[50] C. S. Peskin. The immersed boundary method. Acta Numerica, 11 (2992), 479-517.

[51] K. A. Rejniak, A. R. A. Anderson. A computational study of the development of epithelial acini. I. Sufficient conditions for the formation of a hollow structure. Bull. Math. Biol., to appear.

[52] K. A. Rejniak, A. R. A. Anderson, A computational study of the development of epithelial acini. II. Necessary conditions for structure and lumen stability. Submitted.

[53] M. J. Ardens, A. H. Wyllie. Apoptosis: mechanisms and roles in pathology. International Review of Experimental Pathology, 32 (1991), 223-254.

[54] W. J. Nelson. Adaptation of core mechanisms to generate cell polarity. Nature, 422 (2003), 766-774.

[55] S. C. Ferreira, M. L. Martins, M. J. Vilela. Reaction-diffusion model for the growth of avascular tumor. Physical Review E, 65 (2002) 021907.

[56] A. Patel, E. T. Gawlinski, S. K. Lemieux, R. A. Gatenby. A cellular automaton model of early tumor growth and invasion: the effects of native tissue vascularity and increased anaerobic tumor metabolism. J. Theor. Biol., 213 (2001), 315-331.

[57] A. R. Kansal, Torquato S., E. A. Chiocca, T.S. Deisboeck. Emergence of a subpopulation in a computational model of tumor growth. Journal of Theoretical Biology, 207 (2000), 431-441.

[58] S. Haykin. Neural Networks: a comprehensive foundation, 2nd ed. Prentice Hall, New Jersey, 1999. 
[59] R.M. Sutherland. Cell and environment interactions in tumor microregions: the multicell spheroid model. Science, 240 (1988), 177-184.

[60] M. Höckel, K. Schlenger, S. Höckel, P. Vaupel. Hypoxic cervical cancers with low apoptotic index are highly aggressive. Cancer Res, 59 (1999), No. 18, 4525-4528.

[61] T.G. Graeber, C. Osmanian, T. Jacks, D.E. Housman, Koch C.J., Lowe S.W., A.J. Giaccia. Hypoxia-mediated selection of cells with diminished apoptotic potential in solid tumours. Nature, 379 (1996), 88-91.

[62] C.Y. Kim, M.H. Tsai, C. Osmanian, T.G. Graeber, J.E. Lee, R.G. Giffard, J.A. DiPaolo, D.M. Peehl, A.J. Giaccia. Selection of human cervical epithelial cells that possess reduced apoptotic potential to low-oxygen conditions. Cancer Research, 57 (1997), 4200-4204.

[63] M. Höckel, K. Schlenger, B. Aral, M. Mitze, U. Schaffer, P. Vaupel. Association between tumor hypoxia and malignant progression in advanced cancer of the uterine cervix. Cancer Research, 56 (1996), 4509-4515.

[64] C. E. Shannon. A mathematical theory of information. Bell System Technical Journal, 27 (1948), 379-423.

[65] T. Soussi, G. Lozano. p53 mutation heterogeneity in cancer. Biochemical and Biophysical Research Communications, 331 (2005), 834-842. 